\title{
Pouch of Douglas: A Noble Route for Surgical Specimen Retrieval in Laparoscopic Pelvic Mass Surgery
}

\author{
${ }^{1}$ Abhipsa Mishra, ${ }^{2}$ Sujit Behera
}

\section{ABSTRACT}

Aim: To evaluate the feasibility and surgical outcome of surgical specimen retrieval through the pouch of Douglas by an innovative way of puncturing the same with a $10 \mathrm{~mm}$ trocar and cannula in 100 consecutive women undergoing laparoscopic gynecological procedures for a pelvic mass.

Materials and methods: A prospective study over a period of 2 years from June 2012 to June 2014; 100 cases of pelvic mass (small-to-large) surgeries were done laparoscopically and specimens removed through pouch of Douglas by our own new method of puncturing the same with $10 \mathrm{~mm}$ trocar and cannula and putting the mass in endobag and removing with a grasper. Parameters studied were indications, operative time, blood loss, spillage, postoperative pain, long-term complications.

Results: In $96 \%$ of cases, surgical specimens were retrieved successfully, with minimal spillage without any intraoperative or postoperative complication. Though the rest $4 \%$ were retrieved successfully, $2 \%$ had laceration but they were managed intraoperatively, $2 \%$ had postoperative abscess formation managed conservatively. Only $5 \%$ had pain in vagina at 24 hours on $10 \mathrm{~cm}$ visual analog scale (VAS); $95 \%$ cases had no complaint of dyspareunia on 3rd month follow-up and $5 \%$ were lost to follow-up.

Conclusion: A pouch of Douglas approach for specimen removal by our new method after laparoscopic resection of pelvic masses offers the advantage of less postoperative pain, with minimal spillage, good cosmetic result, and patient satisfaction without prolonging the operative time.

Clinical significance: Tissue retrieved through pouch of Douglas after puncturing with $10 \mathrm{~mm}$ trocar with cannula under vision is a safe, feasible, less time-consuming method in laparoscopic pelvic mass surgery. It avoids the enlargement of operative port site.

Keywords: Incisional hernia, Laparoscopic, NOTES, Port closure.

How to cite this article: Mishra A, Behera S. Pouch of Douglas: A Noble Route for Surgical Specimen Retrieval in Laparoscopic Pelvic Mass Surgery. World J Lap Surg 2018;11(1):29-32.

Source of support: Nil

Conflict of interest: None

\footnotetext{
${ }^{1}$ Assistant Professor, ${ }^{2}$ Consultant

${ }^{1}$ Department of Obstetrics and Gynecology, Kalinga Institute of Medical Sciences, Bhubaneswar, Odisha, India

${ }^{2}$ Department of Obstetrics and Gynecology, Sparsh Hospital \& Critical Care Pvt Ltd, Bhubaneshwar, Odisha, India

Corresponding Author: Abhipsa Mishra, Assistant Professor Department of Obstetrics and Gynecology, Kalinga Institute of Medical Sciences, Bhubaneswar, Odisha, India, e-mail: dramgyn@yahoo.co.in
}

\section{INTRODUCTION}

With the advent of laparoscopic surgery, the major challenge has been to find the easy and safe method of tissue retrieval from the surgical site. Retrieval of small specimen with massive hemoperitoneum and retrieval of medium-to-large specimen sometimes leads to struggle for hours and ultimately it becomes frustrating for the surgeon. The conventional method remains the enlargement of a 5-mm ancillary port-site incision to $10 \mathrm{~mm}$, or more, or through $10 \mathrm{~mm}$ primary port. The use of larger entries does not only implicate cosmetic drawbacks jeopardizing the whole purpose of minimal access surgery but can also increase the chance of injuries involving the inferior epigastric vessels (the most common vascular complication accounting for more than 3 per 1,000 events during operative laparoscopies). ${ }^{1}$ Moreover, enlargement and stretching of port-site incisions have the potential to increase the risk of incisional hernia formation, ${ }^{2}$ postoperative pain, and infection. Whole of the surgeon's effort goes in vein when these complications happen. Removal through pouch of Douglas under vision is one of the natural orifice transluminal endoscopic methods, although this route of specimen extraction has not been explored by many suspicious of expected injury to bowel, bladder, vessel, and dyspareunia. Opening of pouch of Douglas can be done by direct bold incision vaginally or with the help of monopolar hook on the bulging part of vagina after inserting a colpotomizer. We tried a new method of puncturing the pouch of Douglas by $10 \mathrm{~mm}$ trocar cannula under vision at the apex of triangle formed by two uterosacral ligament and retrieved the specimen by tooth grasping forceps (Fig. 1).

\section{MATERIALS AND METHODS}

The study was a prospective study which was conducted in the Department of Gynecology, KIMS Hospital, from June 2012 to June 2014.

\section{Inclusion Criteria}

- Reproductive-age group women (18-45 years)

- Adnexal mass $(3-20 \mathrm{~cm})$

- Benign in nature

Ultrasound investigation was performed before surgery to evaluate the morphology and size of the adnexal mass. 


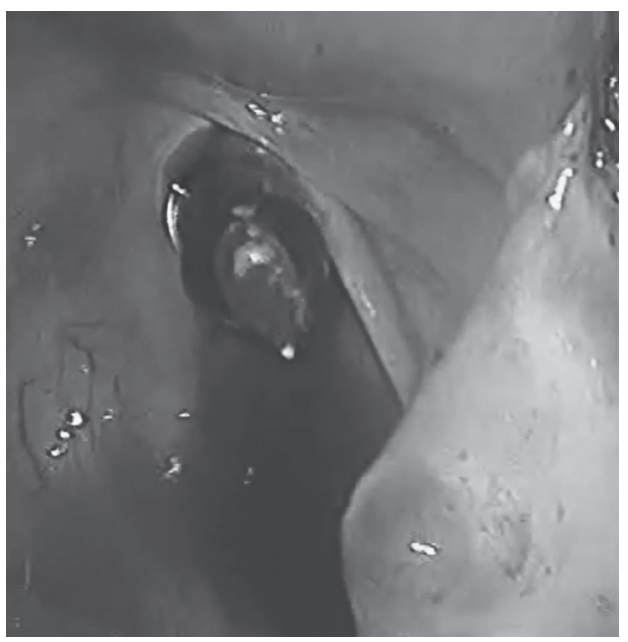

Fig. 1: Puncture of $10 \mathrm{~mm}$ trocar with cannula in pouch of Douglas

Tumor markers were studied in suspected cases and ruled out malignancies.

\section{Exclusion Criteria}

- Unmarried

- Preoperative suspicion or intraoperative diagnosis of malignancy or deep infiltrating endometriosis

- Intraoperative diagnosis of complete obliteration of the pouch of Douglas

- Previous hysterectomy

\section{PROCEDURE}

- Before the procedure, consent was taken from the patient.

- All the surgical procedures were done by the same surgeon and same assistant.

- Injectable third-generation cephalosporin was given just an hour before the procedure.

- General anesthesia was given.

- A $10 \mathrm{~mm}$ supraumbilical primary port and two bilateral $5 \mathrm{~mm}$ side ports were created.

- After complete detachment of the specimen, it was kept inside the endobag.

- A $10 \mathrm{~mm}$ trocar with cannula was punctured in pouch of Douglas just at the apex of triangle made by two uterosacral ligaments under vision, trocar was

Table 1: Patient's characteristics

\begin{tabular}{llll}
\hline Characteristics & Mean & SD & Range \\
\hline Age (years) & 23 & 12 & $18-45$ \\
Body mass index $\left(\mathrm{kg} / \mathrm{m}^{2}\right)$ & 22 & 7 & $16-35$ \\
Adnexa mass size $(\mathrm{cm})$ & 7 & 4 & $3-20$ \\
& No & Percentage \\
Obese (No) & 10 & 10 & \\
Previous abdominal surgery (No) & 20 & 20 & \\
Nulliparous (No) & 15 & 15 & \\
\hline
\end{tabular}

removed and grasping forceps were introduced and held the mouth of endobag and the specimen was removed through pouch of Douglas slowly in sliding manner. Any morcellation was done vaginally.

- Saline lavage was done in all cases after securing hemostasis.

- The colpotomy was closed with a running 0 chromic catgut vaginally.

- Postoperative pain scoring done on 10 cm VAS at 1-, 3-, and 24-hour postoperative period. Postoperative pain was managed with inj dynapar IM 8 hourly for the first 24 hours.

- On discharge, patient was advised abstinence for 6 weeks.

- Follow-up evaluation was scheduled 1 and 3 months after surgery.

\section{PARAMETERS EVALUATED}

- Indications for laparoscopy

- Intraoperative details of the procedure (details of the adnexa mass)

- Time required for surgical specimen removal

- Total operative time

- Estimated blood loss

- Intraoperative and postoperative complications

- Postoperative pain score

\section{Statistical Analysis}

Descriptive statistics were used to analyze the data. Continuous variable results were reported as mean, standard deviation (SD), and range. Categorical data were reported as percentages of the total (Tables 1 to 4 ).

\section{DISCUSSION}

Retrieval of specimen is a big challenge in laparoscopic surgery. Removal of small specimen is not a problem, but removal of medium-to-large specimen leads to struggle for the surgeon. It can be done from the primar port site or enlargement of secondary port site, through a minilaparotomy incision or through pouch of Douglas.

Table 2: Clinical diagnosis

\begin{tabular}{lll}
\hline Characteristic & No & Percentage \\
\hline Simple ovarian cyst & 20 & 20 \\
Hemorrhagic cyst & 10 & 10 \\
Dermoid cyst & 5 & 5 \\
Chocolate cyst & 20 & 20 \\
Hydrosalpinx & 8 & 8 \\
Ectopic pregnancy & 30 & 30 \\
Myoma & 5 & 5 \\
Appendicitis & 2 & 2 \\
\hline
\end{tabular}


Table 3: Laparoscopic procedures and intraoperative details

\begin{tabular}{llll}
\hline $\begin{array}{l}\text { Type of procedure } \\
(\text { Total no }=100)\end{array}$ & No & Percentage & \\
\hline U/L ovarian cystectomy & 15 & 15 & \\
B/L ovarian cystectomy & 5 & 5 & \\
U/L ovariotomy & 5 & 5 & \\
Myomectomy & 5 & 5 & \\
U/L salpingectomy & 20 & 20 & \\
B/L salpingectomy & 8 & 8 & Range \\
M/L salpingo-oophorectomy & 20 & 20 & $10-100$ \\
B/L salpingo-oophorectomy & 10 & 10 & $40-120$ \\
Appendicectomy & 2 & 2 & $5-30$ \\
& Mean & $S D$ \\
Estimated blood loss, mL & 20 & 12 & \\
Operative time, min & 60 & 40 & \\
Specimen retrieval time, min & 15 & 8 & \\
\hline
\end{tabular}

Table 4: Pain score on $10 \mathrm{~cm}$ VAS

\begin{tabular}{lll}
\hline Postoperative time in hours $(1-2 \mathrm{~cm})$ & $N=100$ & Percentage \\
\hline 1 hour & 20 & 20 \\
3 hours & 10 & 10 \\
24 hours & 5 & 5 \\
\hline
\end{tabular}

Removal through primary port needs change of 10 to $5 \mathrm{~mm}$ scope to visualize leads to increase the operative time. Enlargement of port site leads to intraoperative vessel injury and postoperative pain, bad scar, and hernia formation. ${ }^{2-4}$ Minilaparotomy spoils the whole purpose of laparoscopy.

Transvaginal route is a natural route of tissue retrieval explained more than 100 long years back. ${ }^{5}$ Though it has not been explored much by gynecologist in laparoscopic surgery for specimen retrieval in apprehension of potential injury to bowel, bladder, infection, and sexual dysfunction, but nowadays, it has emerged as a preferred site of tissue extraction as a procedure of natural orifice transluminal endoscopic surgery among surgeon. ${ }^{6}$

We tried a new method of opening the pouch of Douglas by puncturing with $10 \mathrm{~mm}$ trocar and cannula with a clean cut margin under vision which avoided use of any colpotomizer or any energy source which may lead to lateral spread to rectum. The advantages of this route are that it is easily stretchable, and drainage of large amount of peritoneal collection is done easily and quickly and closer is easy.

In our study, all the specimens $(100 \%)$ could be removed through the pouch of Douglas. All the masses were removed in endobag without spillage except the specimen of ruptured ectopic. Suction of cyst material was done vaginally. Rapid drainage of blood and clot in massive hemoperitoneum in ruptured ectopic was another advantage of this route. Only two cases had extended laceration of vagina which was sutured intraoperatively and two cases had developed pelvic abscess diagnosed by ultrasound on 1st month followup, managed conservatively with injectable antibiotics. Postoperative $10 \mathrm{~cm}$ VAS score out of 100 in only $5 \%$ had pain $(1-2 \mathrm{~cm})$ at 24 hours; $95 \%$ of the patients had no complaint of dyspareunia on the 3rd month follow-up and $5 \%$ were lost to follow-up.

Studies comparing traditional laparoscopic approaches with transumbilical specimen retrieval vs transvaginal approaches have demonstrated that it is a safe, feasible, and applicable technique. Further research is needed to assess the real advantages of this natural orifice extraction procedure. ${ }^{7}$ Furthermore, studies have demonstrated no increased risk of postoperative infection or incidence of sexual dysfunction or pelvic pain. ${ }^{8}$ Twenty-two women who had undergone laparoscopic posterior colpotomy at initial operative laparoscopy and later underwent a second laparoscopic procedure were evaluated for adhesion formation. It does not appear that tissue removal via laparoscopic colpotomy predisposes reproductive-age women to postoperative adnexal adhesion formation. ${ }^{9}$ Theoretical complications that could be attributed to culdotomy include rectal injury, injury to the bladder and ureters, hemorrhage, vaginal cuff hematoma, vaginal scarring, and postoperative pelvic infections. These complications are rare when the transvaginal route is used. ${ }^{10}$

\section{CONCLUSION}

A pouch of Douglas approach after puncturing with $10 \mathrm{~mm}$ trocar and cannula for specimen removal after laparoscopic resection of pelvic masses offers the advantage of being safe, easy to perform, less time consuming, less postoperative pain, with minimal spillage, good cosmetic result, and patient satisfaction without prolonging the operative time.

\section{REFERENCES}

1. Li TC, Saravelos H, Richmond M, Cooke ID. Complication of laparoscopic pelvic surgery: recognisation, management and preventation. Hum Reprod Update 1997 Sep-Oct;3(5):505-515.

2. Zaki H, Penketh RJ, Newton J. Gynaecological laparoscopy audit: Birmingham experience. Gynaecol Endosc 1995 Jan;4(4):251-257.

3. Boike GM, Miller CE, Spirtos NM, Mercer LJ, Fowler JM, Summitt R, Orr JW Jr. Incisional bowel herniations after operative laparoscopy: a series of nineteen cases and review of the literature. Am J Obstet Gynecol 1995 Jun;172(6): 1726-1733.

4. Kadar N, Reich H, Liu CY, Manko GF, Gimpelson R. Incisional hernias after major laparoscopic gynaecologic procedures. Am J Obstet Gynecol 1993 May;168(5):1493-1495.

5. Ghezzi F, Raio L, Mueller MD, Gyr T, Buttarelli M, Franchi M. Vaginal extraction of pelvic masses following operative laparoscopy. Surg Endosc 2002 Dec;16(12):1691-1696. 
6. Natural Orifice Surgery Consortium for Assessment and Research (NOSCAR ${ }^{\mathrm{TM}}$ ) International conference on natural orifice transluminal endoscopic surgery. New York: NOTES ${ }^{\mathrm{TM}}$. 2005. [cited 2005 Jul]. Available from: http://www.noscar.org.

7. Uccella S, Cromi A, Bogani G, Casarin J, Serati M, Ghezzi F. Transvaginal specimen extraction at laparoscopy without concomitant hysterectomy: our experience and systematic review of the literature. J Minim Invasive Gynecol 2013 SepOct;20(5):583-590.
8. Ghezzi F, Cromi A, Uccella S, Bogani G, Serati M, Bolis P. Transumbilical versus transvaginal retrieval of surgical specimens at laparoscopy: a randomized trial. Am J Obstet Gynecol 2012 Aug;207(2):112.e1-116.e1.

9. Nezhat F, Brill AI, Nezhat CH, Nezhat C. Adhesion formation after endoscopic posterior colpotomy. J Reprod Med 1993 Jul;38(7):534-536.

10. Copenhaver EH. A critical assessment of culdoscopy. Surg Clin North Am 1970 Jun;50(3):713-718. 